\title{
Roles of EBV in Haemopoetic Malignancies
} Eva Klein*‡, Loránd L Kis and Miki Takahara

\author{
Address: Microbiology and Tumor Biology Center (MTC) Karolinska Institutet, 17177 Stockholm Sweden \\ Email: Eva Klein* - eva.klein@mtc.ki.se \\ * Corresponding author $\ddagger$ Presenting author
}

from 2005 International Meeting of The Institute of Human Virology

Baltimore, USA, 29 August - 2 September 2005

Published: 8 December 2005

Retrovirology 2005, 2(SuppI I):S42 doi:I0.II86/I742-4690-2-SI-S42

In addition to the B-cell derived Burkitt lymphoma and the immunoblastomas of immunodefective patients, most of the rare nasal NK lymphomas and about half of the Hodgkin's lymphomas (HL) carry EBV. In the 2 latter malignancies, the neoplastic cells are embedded in granulation tissue (with evidence for the contribution of the microenvironment to the maintenance of the disease). Their viral expression pattern is similar, EBNA-1 and LMP1 pos and EBNA-2 neg (type II). In contrast to the EBVdriven immunoblastomas, EBV cannot be held directly responsible for the growth of these 2 malignancies that also differ among themselves.

\section{Hodgkin's lymphomas}

The few existing HL derived cell lines are EBV negative. When from one line a forcibly converted EBV positive subline was established, it expressed EBNA-1 only (Type I).LMP-1 could be induced by exposure to CD40L and IL4. Conceivably, the in vivo phenotype (Type II) is imposed by the cytokines that abound in the granulation tissue. According to one present view $\mathrm{EBV}$ is important in the early stage of HL development, in that it rescues B lymphocytes with non functional Ig-rearrangements from apoptosis.

\section{Nasal NK/T lymphomas}

Normal NK/T cells do not carry EBV receptors The mechanism of infection of the NK/T cells is not known. EBV carrying cell lines exist and they express the Type II pattern, corresponding thus to the in vivo phenotype. They require IL-2 for in vitro proliferation. Conceivably IL-2 can be provided by activated $\mathrm{T}$ cells in vivo. Treatment with cytokines (IL-10, IFN g) upregulates LMP-1 expression that leads to more efficient growth response to IL-2. 